\title{
Retrospective Data Analysis for Enhanced Recovery after Surgery (ERAS) Protocol for Elderly Patients with Long-Level Lumbar Fusion
}

PENG WANG

Xuanwu Hospital

CHAO KONG

Xuanwu Hospital

ZE TENG

Cancer Hospital Chinese Academy of Medical Sciences

ZHONGEN LI

Xuanwu Hospital

SITAO ZHANG

Xuanwu Hospital

WENZHI SUN

Xuanwu Hospital

Shibao Lu ( $\sim$ shibaoluspine@sina.com )

Xuanwu Hospital of Capital Medical University

\section{Research Article}

Keywords: Enhanced recovery after surgery, Elderly, Lumbar fusion surgery

Posted Date: July 15th, 2021

DOl: https://doi.org/10.21203/rs.3.rs-687347/v1

License: (c) (1) This work is licensed under a Creative Commons Attribution 4.0 International License. Read Full License 


\section{Abstract}

Background: Currently, ERAS for spinal surgery field is still in its beginnings, the major protocol lessons learned from other surgical specialties and lack of ERAS program for elderly patients ( $>70$ years old). Geriatric patients has its own special characteristics resulting in more harmed by surgical stress. The enhanced recovery after surgery (ERAS) are designed to improving recovery after surgery and can result in substantial benefits in both clinical outcomes and cost-effectiveness. In the present study, we aimed to determine whether enhanced recovery after surgery (ERAS) significantly improved satisfaction and outcomes in elderly patients (>70 years old) with long-level lumbar fusion.

Methods: A total of 154 patients were included, 72 in the ERAS group and 82 case-matched patients in the non-ERAS group. Data including demographic, comorbidity and surgical information were collected from electronic medical records. ERAS interventions were categorised as preoperative, intraoperative and postoperative. We also evaluated primary outcome, surgical complication and length of stay (LOS).

Results: There were no statistically significant intergroup differences in regards to demographics, comorbidities, American Society of Anaesthesiologists (ASA) grade, or the number of fusion levels. There were also no differences between mean surgery time of intraoperative blood loss between the ERAS and non-ERAS groups. In addition, the mean preoperative Visual Analogue Score (VAS) for the back and legs and Oswestry Disability Index (ODI) score were not significantly different between the two groups. Overall, ERAS pathway compliance was $91.5 \%$. There were no significant differences in the readmission and mortality rates at 30-day follow-up between the ERAS and non-ERAS groups. However, we observed a statistically significant decrease in the complications in the ERAS group(8 in the ERAS group versus 23 in the non-ERAS group, $p=0.009)$ and LOS in the ERAS group (17.74 \pm 5.56 of ERAS group versus $22.13 \pm 12.21$ in non-ERAS group, $p=0.041)$. Multivariable linear regression showed that comorbidities $(p=0.028)$ and implementation of ERAS program $(p=0.002)$ were correlated with prolonged LOS. Multivariable logistic regression showed that comorbidities $(p=0.029) \otimes$ implementation of ERAS program $(p=0.043)$ and preoperative VAS Back $(p=0.046)$, were correlated with complications.

Conclusions: This report describes the first ERAS protocol used in elderly patients after long-level lumbar fusion surgery. Our ERAS program is safe and could help decreases LOS and complication in elderly patients with long-level lumbar fusion.

\section{Background}

As the medical standards and life expectancy increase, the prevalence of elderly patients with degenerative diseases of the lumbar spine also increases and the proportion of elderly patients presenting for surgery is increasing $(1-3)$. Due to the physiological changes of aging, geriatric patients undergoing spinal surgeries may be different compared to younger patients and usually have more comorbidities, frailty, weakened organ function, etc. Despite the advances in surgical and anesthetic techniques, geriatric patients has undesirable peri-operative complications, morbidity and extended 
convalescence(4). The key pathogenic factor in postoperative morbidity, excluding failures related to surgical or anaesthetic technique, is the surgical stress response(5). All surgical patients inevitably need to face surgical stress response after surgery, an excessive stress response can lead to a pathological state, may predispose the patient to various postoperative complications and the risk of delayed recovery(6). Therefore, reducing stress response can theoretically reduce the occurrence of surgical complications and improve surgical efficacy. Geriatric patients has its own special characteristics resulting in more harmed by surgical stress. The enhanced recovery after surgery (ERAS) are designed to improving recovery after surgery and can result in substantial benefits in both clinical outcomes and costeffectiveness(7). The ERAS principles are meant to reduce stress contains a series of evidence-based protocols for preoperativelintraoperative and postoperative care aims at improve patient satisfaction and faster recovery after surgery, and aims to reduce morbidity, decrease length of hospital stay (LOS). The concept of ERAS was first used in the field of colonic surgery. Although the laparoscopic approach reduction of the stress response to surgery, it is still controversial whether to reduce the postoperative morbidity and LOS(8). Malnutrition $\square$ surgical stress $\square$ intraoperative heat loss $\square$ pain, etc can amplify stress and damage the body. While no single technique or drug regimen has been shown to eliminate postoperative morbidity and mortality, multimodal interventions is necessary to improve the efficacy of surgery(5).

Given the advanced concept of ERAS, ERAS is currently widely used in general surgery(8), urology(9) and gynecology(10). Currently, ERAS for spinal surgery field is still in its beginnings, the major protocol lessons learned from other surgical specialties and lack of ERAS program for elderly patients $(>70$ years old). Xuanwu Hospital of Capital Medical University is China's National Geriatric Clinical Research Center, department of orthopedics performs more than 100 lumbar fusion surgery for more than 70 years old patients per year. Our primary aim of this study was to measure the effectiveness and safety of our ERAS protocol in elderly patients with long-level lumbar fusion. Our secondary aim was to determine which successfully achieved elements are independent predictors of ERAS in patients undergoing long-level lumbar fusion.

\section{Materials And Methods}

Inclusion criteria and patient selection. This is a retrospective cohort study of prospectively collected data. The study enrolled lumbar disc herniation or lumbar spinal stenosis patients if they were over the age of 70 years old underwent lumbar fusion three or more than three levels from July 2019 to June 2021 (ERAS group), and from January 2018 to June 2019 (non-ERAS group). Both groups were cared for by the same surgical team. A retrospective non-ERAS group in which patients were treated under traditional perioperative protocols. Diagnosis of degenerative disorders of the lumbar spine was performed by two spinal orthopaedic specialists based on clinical symptoms and image inspection. Routinely complete the full-length front and side view of the spine before surgery, and measure the sagittal position parameters, coronal parameters, sagittal pelvic parameters. Full-length dynamic position X-ray examination of the spine assesses the stability of the vertebral body in the dynamic position, the flexibility of degenerative scoliosis and the compensatory ability of adjacent segments. In addition, CT three-dimensional 
reconstruction and MRI examination are routinely perfected before surgery to evaluate the bone structure and the degree of compression of the dural sac and nerve root. Surgery was indicated when patients with typical symptoms of spinal stenosis and did not respond to conservative treatments. Individuals who had infection disease, trauma, cauda equina injury, neoplasm were excluded in this study, as well as those planned for a revision of a previous fusion.

Demographic data including age, gender and body mass index (BMI). Comorbidities included hypertension, heart disease, diabetes, osteoporosis, stomach problem, bowel or intestinal problem, psychological symptoms. Other interest included American Society of Anesthesiologists (ASA) physical status score, Oswestry Disability Index (ODI)【Visual Analogue Score (VAS) for back and legs score. Operative records were reviewed to the number of fusion levels, operative time, intraoperative blood loss. The primary outcome data were analyzed included complication, length of stay, postoperative pain scores and 30-day readmission rates. All data were collected from the electronic medical record.

ERAS interventions. ERAS program was proposed and planned in 2017, combined with other ERAS projects and the clinical experience of many experts, our ERAS protocol in elderly patients with long-level lumbar fusion was obtained. The core group consisted of anesthesiologists, spine surgeons, nutritionists, physical therapists, physicians, geriatricians and nurses. With the approval of the ethical committee for human subjects of the Xuanwu Hospital of Capital Medical University ((permit data

2018.4.3; no. 2018086), we began to implement the ERAS program in July 2019. Our ERAS interventions was divided into preoperativelintraoperative and postoperative, included administration of the following: (1) patient education and counseling; (2) no prolonged fasting; (3) carbohydrate drink allowed up to 2 hours before surgery; (4) antithrombotic stockings; (5) antibiotic prophylaxis within 1 hour of incision; (6) tranexamic acid; (7) maintenance of normothermia; (8) local infiltration analgesia; (9) early drinking water after recovery from anesthesialearly feeding started 6 hours; (10) postoperative early ambulation after 48 hours; (11) early removal of bladder catheter after 24 hours; (12) multimodal analgesia. The details of our ERAS were described in our previous study(11).

Statistical analysis. All statistical analyses were performed using the SPSS software version 17.0 (SPSS, Inc., Chicago, IL, USA). Patient demographics, comorbidities data, markers of baseline health, and clinical outcomes were compared between ERAS group and non-ERAS group using Student's test and $\chi 2$ test. Multivariable linear regression analysis was used to assess the association of risk factors (ERAS elements) with LOS.

A value of $P<0.05$ was considered for significant differences.

\section{Results}

Demographics. A total of 154 patients were included, there were 72 patients in the ERAS group (31 men and 41 women, mean age $76.68 \pm 4.83$ years, mean BMI25.50 \pm 3.30 ) and 82 patients in the non-ERAS group ( 26 men and 56 women, mean age $76.38 \pm 4.48$ years, mean BMI $26.05 \pm 3.84$ ). All surgeries were 
performed by a senior surgeon. Preoperative characteristics were similar between the two groups (Table 1). Demographic data were compared, and no statistically significant intergroup differences were observed. And there were not significantly different with comorbidities, ASA grade or the number of fusion levels. The mean ERAS group and non-ERAS group operative time and intraoperative blood loss showed no significant difference. In addition, the mean preoperative JOA, VAS for the back and legs and ODI score showed no significant difference. 
Table 1

Patient demographics

\begin{tabular}{|c|c|c|c|}
\hline Patient Demographics & ERAS & non-ERAS & $\mathbf{P}$ \\
\hline Sample size & 72 & 82 & \\
\hline Age (years) & $76.68 \pm 4.83$ & $76.38 \pm 4.48$ & 0.71 \\
\hline Male/Female & $31 / 41$ & $26 / 56$ & 0.22 \\
\hline Body mass index & $25.50 \pm 3.30$ & $26.05 \pm 3.84$ & 0.91 \\
\hline Smoker & 8 & 10 & 0.84 \\
\hline \multicolumn{4}{|l|}{ Comorbidities } \\
\hline Hypertension & 35 & 44 & 0.53 \\
\hline Heart disease & 12 & 19 & 0.32 \\
\hline Stroke & 3 & 9 & 0.15 \\
\hline Chronic lung disease & 0 & 4 & 0.06 \\
\hline Diabetes & 16 & 18 & 0.97 \\
\hline Osteoporosis & 8 & 13 & 0.39 \\
\hline Gastrointestinal & 3 & 4 & 0.83 \\
\hline Psychological symptoms & 0 & 2 & 0.18 \\
\hline Preoperative ODI, \% & $53.04 \pm 12.36$ & $58.44 \pm 13.32$ & 0.37 \\
\hline Preoperative VAS (Back) & $4.57 \pm 1.85$ & $6.04 \pm 1.30$ & 0.09 \\
\hline Preoperative VAS (Leg) & $5.24 \pm 1.51$ & $6.26 \pm 1.55$ & 0.89 \\
\hline \multicolumn{4}{|l|}{ ASA grade } \\
\hline I & 0 & 0 & \\
\hline II & 42 & 45 & 0.67 \\
\hline III & 30 & 37 & 0.67 \\
\hline IV & 0 & 0 & \\
\hline \multicolumn{4}{|l|}{ No. of levels fusion } \\
\hline 3 & 45 & 59 & 0.85 \\
\hline 4 & 22 & 14 & 0.05 \\
\hline 5 & 5 & 9 & 0.78 \\
\hline Operative time & $283.87 \pm 57.75$ & $275.94 \pm 60.51$ & 0.95 \\
\hline
\end{tabular}




\begin{tabular}{|llll|}
\hline Patient Demographics & ERAS & non-ERAS & P \\
\hline Intraoperative blood loss & $518.20 \pm 231.12$ & $605.41 \pm 273.81$ & 0.11 \\
\hline Intraoperative blood transfusion & $485.76 \pm 395.50$ & $499.58 \pm 374.55$ & 0.35 \\
\hline
\end{tabular}

Compliance to ERAS pathway. Our ERAS protocol included 12 elements interventions (Table 2), overall pathway compliance was $91.5 \%$ (Table 2). Patient education and counselling, no prolonged fasting, antithrombotic stockings, antimicrobial prophylaxis, all Intra-operative ERAS items and Perioperative multimodal analgesia were used in $100 \%$ of cases. Items with the lowest compliance were early ambulation (53.7\%).

Table 2

ERAS pathway compliance

\begin{tabular}{|ll|}
\hline \multicolumn{2}{|l|}{ Compliance with the ERAS program } \\
\hline Variable & $\mathrm{n}(\%)$ \\
\hline Pre-operative ERAS items & \\
\hline Patient education and counselling & $72(100)$ \\
\hline No prolonged fasting & $72(100)$ \\
\hline Fluid and carbohydrate loading & $70(97)$ \\
\hline Antithrombotic stockings & $72(100)$ \\
\hline Antimicrobial prophylaxis & $72(100)$ \\
\hline Intra-operative ERAS items & \\
\hline Tranexamic acid & $72(100)$ \\
\hline Maintenance of normothermia & $72(100)$ \\
\hline Local infiltration analgesia & $72(100)$ \\
\hline Postoperative ERAS items & \\
\hline Early oral feeding & $67(93.5)$ \\
\hline Early ambulation & $39(53.7)$ \\
\hline Early removal of bladder catheter & $41(56.5)$ \\
\hline Multimodal analgesia & $72(100)$ \\
\hline Overall compliance (rate) & 91.5 \\
\hline
\end{tabular}

Outcomes. The main clinical outcomes are shown in Table 3, after the implementation of ERAS, there was no significant difference in readmission and mortality at 30-day follow-up between ERAS group and 
non-ERAS group, as complete data were available for $91 \%$ of patients at this early time point. However, we observed a statistically significant decrease in complications in the ERAS group(6 of ERAS group versus 23 in non-ERAS group, $p=0.006)$ and LOS in the ERAS group (17.74 \pm 5.56 of ERAS group versus $22.13 \pm 12.21$ in non-ERAS group, $p=0.041$ ). Multivariable linear regression showed that comorbidities $(p$ $=0.028)$ and implementation of ERAS program $(p=0.002)$ were correlated with prolonged LOS. On the other hand, age $(p=0.94)$, sex $(p=0.649), \operatorname{BMI}(p=0.535)$, smoker $(p=0.137), A S A \geq 3(p=0.062)$, fusion number $(p=0.537)$, operative time $(p=0.748)$, blood loss $(p=0.235)$, preoperative VAS Back $(p=0.667)$, preoperative VAS Leg $(p=0.062)$ and preoperative ODI $(p=0.831)$ were not related to LOS.

Table 3

Postoperative outcomes

\begin{tabular}{|llll|}
\hline Outcome measure & ERAS & non-ERAS & P \\
\hline Complications & 8 & 23 & 0.009 \\
\hline Bronchial aspiration & 0 & 0 & \\
\hline Hypoproteinemia & 2 & 5 & \\
\hline Electrolyte disturbance & 2 & 4 & \\
\hline Heart disease & 1 & 3 & \\
\hline Cerebrovascular accident & 0 & 1 & \\
\hline Pneumonia & 1 & 5 & \\
\hline Urinary tract infection & 1 & 2 & \\
\hline Surgical site infection & 0 & 3 & \\
\hline Deep vein thrombosis & 0 & 2 & \\
\hline Spinal fluid leakage & 1 & 1 & \\
\hline Neurological & 0 & 0 & \\
\hline Fall down & 0 & 0 & \\
\hline LOS & $17.74 \pm 5.56$ & $22.13 \pm 12.21$ & \\
\hline 30-day readmissions & 0 & 1 & \\
\hline 30-day mortality & 0 & 0 & \\
\hline
\end{tabular}


Table 4

Multivariable analyses for LOS and complications

\begin{tabular}{|c|c|c|c|c|}
\hline \multirow[t]{2}{*}{ characteristic } & \multicolumn{2}{|c|}{$\begin{array}{l}\text { Multivariable linear regression } \\
\text { for LOS }\end{array}$} & \multicolumn{2}{|c|}{$\begin{array}{l}\text { Multivariable logistic regression for } \\
\text { complications }\end{array}$} \\
\hline & Coefficient $(95 \% \mathrm{Cl})$ & value & OR $(95 \% \mathrm{Cl})$ & $\mathrm{p}$ value \\
\hline Age & $0.12(-0.4$ to 0.6$)$ & 0.94 & $1.075(0.944-1.224)$ & 0.275 \\
\hline Female & $2.6(-1.042$ to 6.5$)$ & 0.649 & $2.5(0.439-14.233)$ & 0.302 \\
\hline BMI & $0.186(-0.325$ to 0.697$)$ & 0.535 & $1.022(0.87-1.2)$ & 0.792 \\
\hline Smoker & $0.76(-0.21$ to 1.11$)$ & 0.137 & $2.10(0.85-3.24)$ & 0.121 \\
\hline Comorbidities & $1.26(0.29$ to 2.23$)$ & 0.028 & $2.35(1.089-5.077)$ & 0.029 \\
\hline$A S A \geq 3$ & $0.98(-0.03$ to 1.92$)$ & 0.062 & $2.31(0.98-4.53)$ & 0.060 \\
\hline Fusion number & $\begin{array}{l}-0.832(-3.572 \text { to } \\
1.908)\end{array}$ & 0.537 & $0.723(0.307-1.702)$ & 0.457 \\
\hline Operative time & $0.379(-2.03$ to 2.78$)$ & 0.748 & $0.675(0.31-1.42)$ & 0.304 \\
\hline Blood loss & $0.004(-0.002$ to 0.007$)$ & 0.235 & $1.0(0.98-1.01)$ & 0.665 \\
\hline ERAS & $-2.98(-4.1$ to -1.00$)$ & 0.002 & $0.29(0.1-0.9)$ & 0.043 \\
\hline $\begin{array}{l}\text { Preoperative VAS } \\
\text { (Back) }\end{array}$ & $0.293(-1.095$ to 1.68$)$ & 0.667 & $1.742(1.01-3)$ & 0.064 \\
\hline $\begin{array}{l}\text { Preoperative VAS } \\
\text { (Leg) }\end{array}$ & $-0.929(-1.907$ to 0.05$)$ & 0.062 & $1.3(0.95-2.9)$ & 0.082 \\
\hline $\begin{array}{l}\text { Preoperative ODI } \\
(\%)\end{array}$ & $\begin{array}{l}1.325(-11.346 \text { to } \\
13.697)\end{array}$ & 0.831 & $1.36(0.74-2.28)$ & 0.307 \\
\hline
\end{tabular}

Multivariable logistic regression showed that comorbidities $(p=0.029)$, implementation of ERAS program $(p=0.043)$ and preoperative VAS Back $(p=0.046)$, were correlated with complications. On the other hand, age $(p=0.275)$, sex $(p=0.302)$, BMI $(p=0.792)$, smoker $(p=0.121), A S A \geq 3(p=0.060)$, fusion number ( $=0.457)$, operative time $(p=0.304)$, blood loss $(p=0.665)$, preoperative VAS Leg $(p=0.082)$ and preoperative ODI $(p=0.307)$ were not related to complications.

\section{Discussion}

We found that the use of the our ERAS protocol was beneficial and safe for elderly patients ( $>70$ years old) undergoing long-level lumbar fusion surgery. For ERAS group, both the complications and LOS were lower in comparison with control group and without increasing 30-day readmission and mortality rates. We also found that fewer comorbidities and ERAS were associated with incrementally improved complication odds as well as reduced length of stay. 
Due to reduced physiologic reserve of vital organs, elderly patients are usually accompanied by significant comorbidities. Therefore, generally considered a minor complication in younger adult patients and may produce only transient adverse effects, may have much more severe consequences in the elderly patients and result in a significantly prolonged LOS(12).

Symptoms of patients with multi-segment degeneration of the lumbar spine often manifest as low back pain and lower limb radiculopathy, which can be accompanied by various degrees of lumbar spinal stenosis, intervertebral disc herniation, scoliosis, and lumbar spondylolisthesis, and often involve multiple segments. Often involves multi-level decompression fusion and internal fixation, so the operation is more difficult, the perioperative risk is greater. Due to the long-level lumbar fusion destroys more paravertebral muscles and facet joints, theoretically, the operation time is longer, the amount of bleeding is more, the risk of potential dural nerve injury is higher and postoperative complications are relatively high.

The key parts of the concept of ERAS include reducing the physical traumatic stress and accelerate rehabilitation, which is of great significance for long-level lumbar fusion. We assessed the association between the level of ERAS component implementation and perioperative complications, length of stay, and overall hospitalization costs. This was based on eight core ERAS protocol components.

The negative effects of traditional kept in bed after surgery may not only increase the risk of thromboembolic and pulmonary complications, but also increased loss of muscle tissue(5). Early mobilization is regarded as a key component of ERAS, consistent with the goals of supporting the early reestablishment of normal function(13). It's been demonstrated that early ambulation after long-level lumbar surgery significantly reduces the incidence of postoperative complications includes pulmonary complications, thromboembolism and shortens the LOS. $(14,15)$. Despite the known benefits of early mobility, there is little guidance available on how soon among uncomplicated after long-level lumbar surgery over 70 years old patients should get out of bed and ambulate. Compared with the previous shortlevel lumbar fusion patients over 65 years old(16), for patients over 70 years old, which have worse physical function, more fusion segments and greater surgical trauma, contribute to the risk for adverse outcomes. Given the complexity of this population, we have extended the patient ambulate and remove the catheter time to 72 hours, in-bed active/passive limb movement within 4 hours after surgery. Our research results show that this improvement is safe and effective in improving the efficacy of long-level lumbar fusion surgery.

In addition to muscle wasting, postoperative high catabolism is also an important factor affecting the overall recovery, catabolism is mediated by the surgical stress and postoperative starvation(5). Our ERAS protocols combine many different elements of care in the preoperative, intraoperative and postoperative periods, and aim to reduce surgical stress, the focus is on early recovery, nutrition and pain relief.

Traditional preoperative fasting for at least $8 \mathrm{~h}$ and oral feeding on postoperative 1 day were considered to be a necessary to reduce the risk of aspiration during anesthesia induction(17), and ameliorate the postoperative nausea/ vomiting(18). However, a number of studies has been shown that traditionally preoperative and postoperative fasting care may cause the postoperative insulin resistance and 
electrolyte disorders $(19,20)$. Clinical observational studies have demonstrated that perioperative hyperglycemia increase the incidence of the development of postoperative complications and death in diabetic and non-diabetic patients(21). In addition, substantial evidence has shown that postoperative electrolyte disorders complications are associated with longer LOS and increased hospital costs in addition to complications such as delirium(22), thromboembolism(23), cardiac and cerebral dysfunction $(12,24,25)$. This in turn, shorting fasting and feeding time help reduce catabolism and loss of muscle tissue and function may include stress reduction, decrease the feeling of hunger and anxiety, improve patient comfort $(26,27)$. Furthermore, avoidance of prolonged preoperative fasting and oral carbohydrate loading can create a positive impact on perioperative glucose control and muscle preservation after major operations(28). One study demonstrated that the mechanism may induces endoplasmic reticulum stress and generates insulin resistance in the skeletal muscle through suppression of Glut4 and inactivation of $\mathrm{Ca} 2+-$ ATPase, leading to intracellular calcium homeostasis disruption and peripheral insulin resistance.(27). Our studies showed that elderly patients ( $>70$ years old) who have undergone long-level lumbar surgery minimizing the duration of fasting in surgical patients preand post-surgery is safe and can effectively low down the incidence of electrolyte disorders.

However, this study has several limitations. This study is the retrospective design, small sample size. The observation time was limited to the hospitalization period, given the lack of long-term follow-up data, definitive conclusions was unknown. Furthermore, the ERAS and non-ERAS group were assessed at different times, which may have introduced analytical bias. Further multicenter studies with a larger participant population and long-term follow-up are required to confirm the safety and efficacy of our ERAS protocol in elderly patients after long-level lumbar fusion surgery.

\section{Conclusions}

In conclusion, this report describes the first ERAS protocol used in elderly patients with long-level lumbar fusion surgery. Our ERAS program is safe and associated with incremental benefits in respect to complications and LOS with long-level lumbar fusion. While still in its infancy, with modified approaches to our ERAS protocol, will likely improve satisfaction and outcomes.

\section{Abbreviations}

ERAS, enhanced recovery after surgery; LOS, length of stay; BMI, body mass index; ASA, American Society of Anesthesiologists; ODI, Oswestry Disability Index; VAS, Visual Analogue Score

\section{Declarations}

\section{Ethics approval and consent to participate}

The study protocol was approved by the ethical committee for human subjects of the Xuanwu Hospital of Capital Medical University 


\section{Consent for publication}

Not applicable

\section{Availability of data and materials}

All data generated or analyzed during this study are included in this published article

\section{Competing interests}

The authors declare no financial and non-financial competing interests.

\section{Funding}

This work was supported by the Beijing Municipal Health Commission (Jing2019-2).

\section{Authors' contributions}

P.W., C.K., and S.L. conceived of and designed the experiments. P.W., C.K., and TZ. performed the experiments. Z.L., S.Z. and W.S. analysed the data. P.W. wrote the paper. S.L. revised the manuscript. All authors reviewed the manuscript.

\section{Acknowledgements}

Not applicable.

\section{References}

1. Fehlings MG, Tetreault L, Nater A, Choma T, Harrop J, Mroz T, et al. The Aging of the Global Population: The Changing Epidemiology of Disease and Spinal Disorders. Neurosurgery. 2015;77(Suppl 4):1-5.

2. Saleh A, Thirukumaran C, Mesfin A, Molinari RW. Complications and readmission after lumbar spine surgery in elderly patients: an analysis of 2,320 patients. Spine J. 2017;17(8):1106-12.

3. Rihn JA, Hilibrand AS, Zhao W, Lurie JD, Vaccaro AR, Albert TJ, et al. Effectiveness of surgery for lumbar stenosis and degenerative spondylolisthesis in the octogenarian population: analysis of the Spine Patient Outcomes Research Trial (SPORT) data. J Bone Joint Surg Am. 2015;97(3):177-85.

4. Lees MC, Merani S, Tauh K, Khadaroo RG. Perioperative factors predicting poor outcome in elderly patients following emergency general surgery: a multivariate regression analysis. Can J Surg. 2015;58(5):312-7.

5. Kehlet $\mathrm{H}$. Multimodal approach to control postoperative pathophysiology and rehabilitation. $\mathrm{Br} \mathrm{J}$ Anaesth. 1997;78(5):606-17.

6. Ren L, Zhu D, Wei Y, Pan X, Liang L, Xu J, et al. Enhanced Recovery After Surgery (ERAS) program attenuates stress and accelerates recovery in patients after radical resection for colorectal cancer: a 
prospective randomized controlled trial. World J Surg. 2012;36(2):407-14.

7. Staartjes VE, de Wispelaere MP, Schroder ML. Improving recovery after elective degenerative spine surgery: 5-year experience with an enhanced recovery after surgery (ERAS) protocol. Neurosurg Focus. 2019;46(4):E7.

8. Meillat H, Brun C, Zemmour C, de Chaisemartin C, Turrini O, Faucher M, et al. Laparoscopy is not enough: full ERAS compliance is the key to improvement of short-term outcomes after colectomy for cancer. Surg Endosc. 2020;34(5):2067-75.

9. Una Orejon R, Mateo Torres E, Huercio Martinez I, Jofre Escudero C, Gomez Rivas J, Diez Sebastian J, et al. Application of ERAS (Enhanced Recovery After Surgery) and laparoscopic surgery in the management of patients with bladder cancer. Arch Esp Urol. 2018;71(2):178-86.

10. Scheib SA, Thomassee M, Kenner JL. Enhanced Recovery after Surgery in Gynecology: A Review of the Literature. J Minim Invasive Gynecol. 2019;26(2):327-43.

11. Li ZE, Lu SB, Kong C, Sun WZ, Wang P, Zhang ST. Impact of Compliance with an Enhanced Recovery After Surgery Program on the Outcomes Among Elderly Patients Undergoing Lumbar Fusion Surgery. Clin Interv Aging. 2020;15:2423-30.

12. Rajpal S, Lee Nelson E, Villavicencio AT, Telang J, Kantha R, Beasley K, et al. Medical complications and mortality in octogenarians undergoing elective spinal fusion surgeries. Acta Neurochir (Wien). 2018;160(1):171-9.

13. Castelino T, Fiore JF Jr, Niculiseanu P, Landry T, Augustin B, Feldman LS. The effect of early mobilization protocols on postoperative outcomes following abdominal and thoracic surgery: A systematic review. Surgery. 2016;159(4):991-1003.

14. Adogwa O, Elsamadicy AA, Fialkoff J, Cheng J, Karikari IO, Bagley C. Early Ambulation Decreases Length of Hospital Stay, Perioperative Complications and Improves Functional Outcomes in Elderly Patients Undergoing Surgery for Correction of Adult Degenerative Scoliosis. Spine (Phila Pa 1976). 2017;42(18):1420-5.

15. Rupich K, Missimer E, O'Brien D, Shafer G, Wilensky EM, Pierce JT, et al. The Benefits of Implementing an Early Mobility Protocol in Postoperative Neurosurgical Spine Patients. Am J Nurs. 2018;118(6):46-53.

16. Wang P, Wang Q, Kong C, Teng Z, Li Z, Zhang S, et al. Enhanced recovery after surgery (ERAS) program for elderly patients with short-level lumbar fusion. J Orthop Surg Res. 2020;15(1):299.

17. Dilmen OK, Yentur E, Tunali Y, Balci H, Bahar M. Does preoperative oral carbohydrate treatment reduce the postoperative surgical stress response in lumbar disc surgery? Clin Neurol Neurosurg. 2017;153:82-6.

18. Steenhagen E. Enhanced Recovery After Surgery: It's Time to Change Practice! Nutr Clin Pract. 2016;31(1):18-29.

19. Xu B, Xu WX, Lao YJ, Ding WG, Lu D, Sheng HF. Multimodal Nutritional Management in Primary Lumbar Spine Surgery: A Randomized Controlled Trial. Spine (Phila Pa 1976). 2019;44(14):967-74. 
20. Cao G, Huang Q, Xu B, Huang Z, Xie J, Pei F. Multimodal Nutritional Management in Primary Total Knee Arthroplasty: A Randomized Controlled Trial. J Arthroplasty. 2017;32(11):3390-5.

21. Kwon S, Thompson R, Dellinger P, Yanez D, Farrohki E, Flum D. Importance of perioperative glycemic control in general surgery: a report from the Surgical Care and Outcomes Assessment Program. Ann Surg. 2013;257(1):8-14.

22. Fineberg SJ, Nandyala SV, Marquez-Lara A, Oglesby M, Patel AA, Singh K. Incidence and risk factors for postoperative delirium after lumbar spine surgery. Spine (Phila Pa 1976). 2013;38(20):1790-6.

23. Fineberg SJ, Oglesby M, Patel AA, Pelton MA, Singh K. The incidence and mortality of thromboembolic events in lumbar spine surgery. Spine (Phila Pa 1976). 2013;38(13):1154-9.

24. Fineberg SJ, Oglesby M, Patel AA, Singh K. Incidence and mortality of perioperative cardiac events in cervical spine surgery. Spine (Phila Pa 1976). 2013;38(15):1268-74.

25. Marquez-Lara A, Nandyala SV, Fineberg SJ, Singh K. Cerebral vascular accidents after lumbar spine fusion. Spine (Phila Pa 1976). 2014;39(8):673-7.

26. Canbay O, Adar S, Karagoz AH, Celebi N, Bilen CY. Effect of preoperative consumption of high carbohydrate drink (Pre-Op) on postoperative metabolic stress reaction in patients undergoing radical prostatectomy. Int Urol Nephrol. 2014;46(7):1329-33.

27. Lin MW, Chen $\mathrm{Cl}$, Cheng TT, Huang CC, Tsai JW, Feng GM, et al. Prolonged preoperative fasting induces postoperative insulin resistance by ER-stress mediated Glut4 down-regulation in skeletal muscles. Int J Med Sci. 2021;18(5):1189-97.

28. Pogatschnik C, Steiger E. Review of Preoperative Carbohydrate Loading. Nutr Clin Pract. 2015;30(5):660-4. 